\title{
Bound-exciton-induced current bistability in a silicon light-emitting diode
}

\author{
J. M. Sun, ${ }^{\text {a) }}$ T. Dekorsy, W. Skorupa, B. Schmidt, and M. Helm \\ Institute of Ion Beam Physics and Materials Research, Forschungszentrum Rossendorf, P.O. Box 510119, \\ 01314 Dresden, Germany
}

\begin{abstract}
A bound-exciton-induced current bistability is observed under forward bias in an efficient silicon light-emitting diode at low temperatures. Two stable states of the S-type differential conductivity correspond to empty and filled states of bound excitons, respectively. The relationship between the current-voltage characteristic and the bound-exciton population can be accounted for using a rate equation model for bound and free excitons. The consistency between the theoretical and experimental results indicates that bound excitons, despite their neutral-charged states, contribute to the current bistability in silicon $p-n$ junction diodes.
\end{abstract}

The bistable $I-V$ characteristics of a semiconductor device usually manifest in a hysteretic switching between a high-impedance, low-current (off) state and a lowimpedance, high-current (on) state. ${ }^{1}$ This nonlinear transport phenomenon has been the basis of a family of static random access memories ${ }^{2}$ logic circuits, ${ }^{3,4}$ as well as high-frequency oscillators. ${ }^{5,6}$ A classical process leading to an S-shaped (bistable) $I-V$ characteristic is related to impact ionization of shallow impurities at low temperatures in bulk semiconductors. ${ }^{7,8}$ Other types of bistable $I-V$ characteristics were usually observed in semiconductor structures with at least one potential barrier for electronic transport, such as metal-insulator switches, ${ }^{9}$ doping superlattices, ${ }^{10}$ and double-barrier resonant-tunneling diodes. ${ }^{11,12}$ Although the conditions or origins leading to the bistability might differ for various device structures, it is commonly assumed that the charge accumulation at or the release from the potential wells in the structures causes a change of the band bending of the potential barriers, and subsequently, the electrostatic feedback leads to the bistability in the $I-V$ characteristics. ${ }^{1}$ Exciton-induced optical bistability is well known in III-V semiconductors. However, there have been very few studies on the exciton-induced current bistability in heterostructures. ${ }^{13}$ The reason is probably that excitons cannot cause a band bending and redistribution of charge profiles due to their intrinsically neutral-charged states. In this report, we present the observation of an S-type differential resistance in the $I-V$ characteristics of a silicon $p-n$ diode produced by boron ion implantation. Similar devices have recently attracted large interest as efficient silicon based light-emitting diodes. ${ }^{14}$ The current bistability in our device is based on the formation and ionization of excitons bound to the shallow traps introduced by boron ion implantation and subsequent high-temperature annealing, as determined by the simultaneous measurement of the bistable current and the excitonic electroluminescence (EL) as a function of the applied voltage. Our model gives a complete description of the origin of the S-type negative differential resistance.

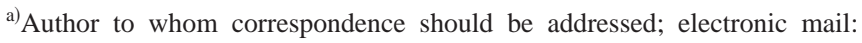
J.Sun@fz-rossendorf.de
}

The silicon $p-n$ diode was prepared by boron implantation into (100) oriented $n$-type $(0.1 \Omega \mathrm{cm})$ silicon substrates with a dose of $4 \times 10^{15} \mathrm{~cm}^{-2}$ at an energy of $25 \mathrm{keV}$ through a 50-nm thermally grown $\mathrm{SiO}_{2}$ layer. The sample was subsequently annealed at $1050^{\circ} \mathrm{C}$ for $20 \mathrm{~min}$ and processed into 1 -mm-diameter diodes (active area $0.00785 \mathrm{~cm}^{2}$ ). The diode was mounted on the cold finger of a closed-cycle cryostat by silver paste for low-temperature EL studies. $I-V$ characteristics were measured with a sourcemeter (Keithley 2410) in a current- or voltage-controlled mode. EL spectra were measured with a monochromator and a liquid nitrogen cooled InGaAs detector. The EL peak intensity was recorded simultaneously with the $I-V$ characteristics.

The diode shows efficient room-temperature EL from band-edge electron-hole recombination of silicon with an external quantum efficiency up to $0.1 \%$. EL spectra are measured at $12 \mathrm{~K}$ for different injection currents, as shown in Fig. 1. The EL spectra show three bands, marked as P1, P2, and P3. The band P1 $(1.1 \mathrm{eV})$ is due to TO phonon-assisted recombination of free excitons in silicon. The spectral shape and the peak energy of P2 are similar to the photoluminescence band from a boron doped $p^{+}$silicon wafer with a hole concentration of $3 \times 10^{18} \mathrm{~cm}^{-3}$. ${ }^{15}$ It is therefore assigned to TO phonon-assisted recombination of the bound excitons

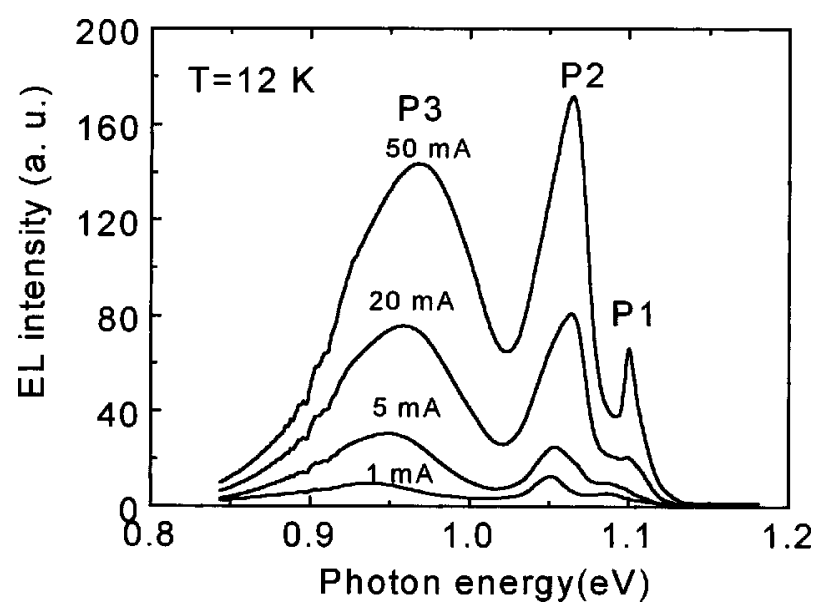

FIG. 1. EL spectra of a silicon $p-n$ diode at a temperature of $12 \mathrm{~K}$ with different injection currents. 


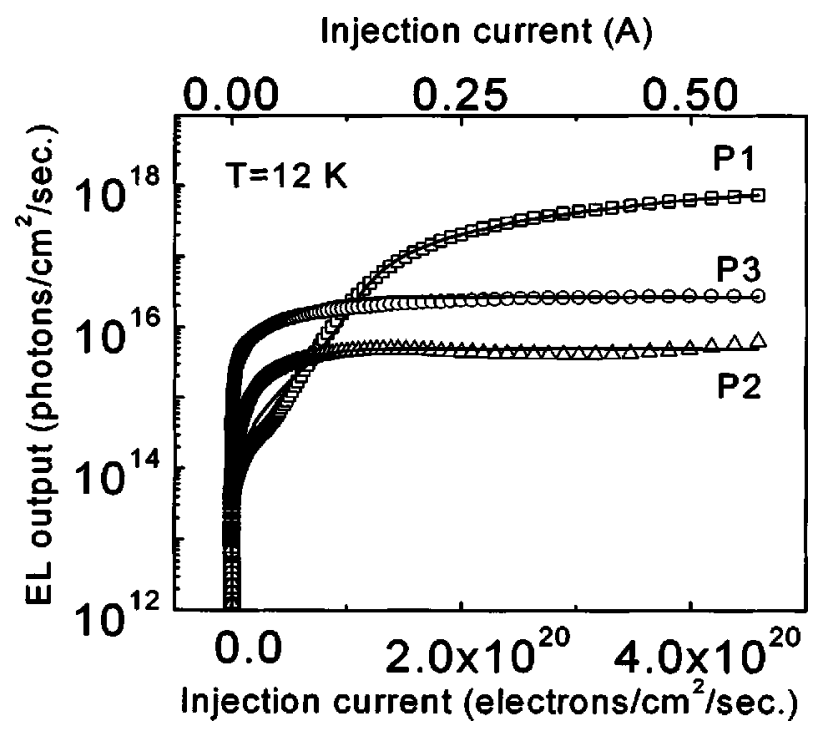

FIG. 2. Dependence of EL output photons as a function of the injection current for different emission peaks P1 (open squares), P2 (open triangles), and P3 (open circles) measured at $12 \mathrm{~K}$. The scattered plots are experimental data, and the solid lines represent a fit with using the rate equation model.

around the localized $p^{+}$region close to the $p-n$ junction. The broad band P3 at $0.9-1.0 \mathrm{eV}$ is probably related to the strain-induced reduction of the band gap in combination with the confinement of carriers by localized electric fields due to high boron concentrations around the dislocations. ${ }^{16} \mathrm{~A}$ detailed study of the two trap bands (P2 and P3) will be given in a forthcoming paper. The dependences of the integrated EL intensity as a function of injection current for the three peaks are shown in Fig. 2. Initially, only the trap bands P2 and P3 are observed in the EL spectra at low current injection. Then, the EL intensity of P2 and P3 saturate with increasing current. Finally, after saturation of the shallower trap band P2, the free exciton peak P1 emerges and its intensity grows linearly with increasing current.

The $I-V$ characteristics of the diode show an S-type

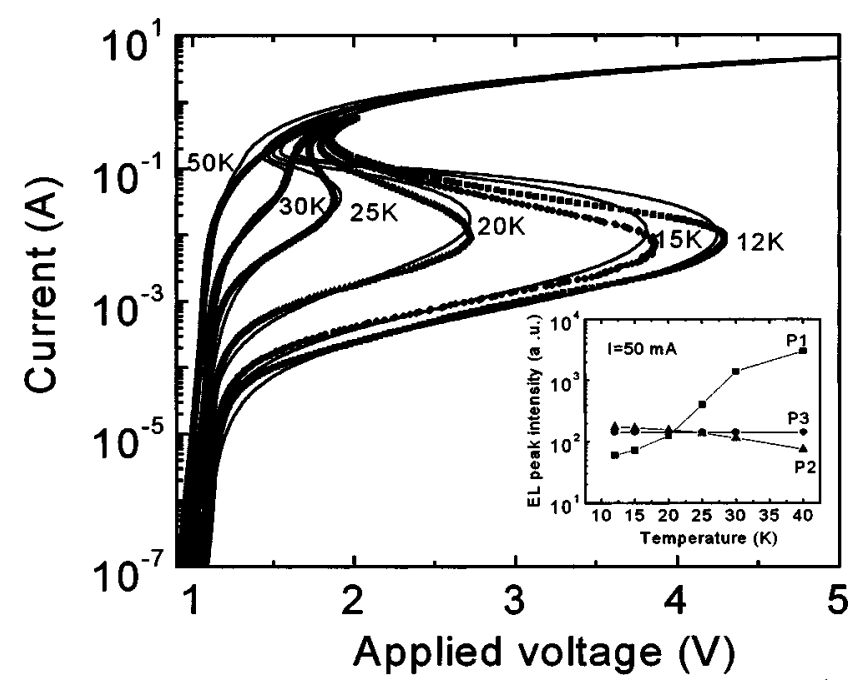

FIG. 3. Current-controlled $I-V$ characteristics of the silicon $p-n$-junction diode at different temperatures. The scattered plots are experimental data and the solid lines represent calculations. Note that the high-current branch can only be measured up to $600 \mathrm{~mA}$ due to the high dissipated power. The insert shows the temperature dependence of the EL intensity at different peaks. negative resistance characteristic in a current-controlled sweeping mode at temperatures ranging from 12 to $30 \mathrm{~K}$, as shown in Fig. 3. The negative resistance region starts from a low-current stable state at the emergence of $\mathrm{P} 2$ and ends at a high-current stable state where the EL of the shallower trap band P2 saturates. The typical current contrast between the two stable branches is of the order of $10^{4}$. The high and the low current states can be well fitted by simply setting a small and a large value to the series resistance $R_{s}$, in the equation of a $p-n$ junction diode, $J=J_{0}\left[\exp \left(V_{j}-J \cdot R_{s}\right) /(n k T)-1\right]$, where $V_{j}, J$, and $T$ are the applied voltage, current, and the temperature, respectively, and $J_{0}, n$, and $k$ are constants. At $50 \mathrm{~K}$, the S-type negative resistance should be thermally quenched through a decrease of the series resistance $R_{s}$ of the lower current state due to increasing free-carrier emission from the shallow traps. Since no obvious overall shift of the $I-V$ characteristics of the $p-n$ junction is observed with increasing current, the shallow traps, which contribute to the negative resistance at low temperatures, must be neutralcharged traps such as excitonic traps, neutral donors, or acceptors. In order to determine which trap band contributes to the negative resistance, the temperature dependences of the peak intensity of $\mathrm{P} 1, \mathrm{P} 2$, and $\mathrm{P} 3$ are measured at a fixed current of $50 \mathrm{~mA}$, as shown in the insert of Fig. 3. The thermal emission of the free carriers from the shallower excitonic traps is confirmed by an increase of P1 and the simultaneous decrease of $\mathrm{P} 2$ with increasing temperature. The EL intensity of P3 is constant in this temperature range due to insufficient thermal energy for ionization of deeper traps. These results indicate that the shallower bound excitons at least partially contribute to the negative differential resistance of the $p-n$ diode.

This interpretation of the observed correlation between carrier transport and EL can be fully accounted for by a simplified system containing free and bound excitons. ${ }^{17}$ Assuming that the minority free-carrier concentration is proportional to the free-exciton concentration with a thermal equilibrium constant $\gamma$, the relationships between the EL intensity of the free (bound) excitons and the injection current can be simulated by solving the steady-state solutions of three rate equations for the number of free excitons, the shallower bound excitons $\mathrm{P} 2$, and traps $\mathrm{P}$, taking into account all the ionization, capture, and recombination processes. The solutions are shown in Fig. 2. The series resistance $R_{s}$ of the diode can be expressed as a function of the mean filling factor $f_{t}$ of bound exciton $\mathrm{P} 2$ and trap P3:

$$
R_{s} \approx R_{c}+\frac{1-f_{t}}{e \cdot\left(\mu_{e}+\mu_{h}\right)\left[\left(\gamma \alpha-n_{0 b}\right) \cdot f_{t}+n_{0 b}\right]},
$$

where $R_{c}$ is a constant small resistance related to the ohmic contact of the electrode; $\mu_{e}\left(\mu_{h}\right)$ and $e$ are the mobilities of free electrons (holes) and electron charge, respectively; $n_{0 b}$ is the background free-carrier concentration at zero current; $\alpha$ is a parameter determined by $\alpha=N_{t}\left(e_{t}+W_{t}\right) / c_{t}$, where $N_{t}, e_{t}, W_{t}$, and $c_{t}$ are the sum of the trap densities, the average thermal emission, recombination, and capture rate, respectively, of bound exciton P2 and trap P3. Equation (1) shows that $\alpha$ and $n_{0 b}$ are key parameters for the observation of the negative resistance; that is the second term of the right side of Eq. (1) would decrease dramatically with the increas- 
ing filling factor $f_{t}$ of the trap states at a low temperature when $n_{0 b} \ll \gamma \alpha$. The series resistance $R_{s}$ is reduced from a high stable value $\sim 1 /\left[e \cdot\left(\mu_{e}+\mu_{h}\right) n_{0 b}\right]$ to a low stable value close to $R_{c}$ as $f_{t}$ varies from 0 to 1 with increasing the injection current; this is the origin of the negative resistance at low temperature. As the temperature increases, thermal ionization of the shallower excitonic traps as well as the neutral acceptors (donors) causes a strong increase of $n_{0 b}$ in the diode. Finally, the series resistance is reduced to a single value $\sim R_{c}+1 /\left[e \cdot\left(\mu_{e}+\mu_{h}\right) n_{0 b}\right]$ for $n_{0 b} \gg \gamma \alpha$, where the capture and emission of free carriers by traps has a weak influence on the series resistance of the diode. Therefore, thermal quenching of the negative resistance can be observed at high temperature, as shown in Fig. 3. The S-type $I-V$ curves are also calculated at different temperature in Fig. 3 by the model. The theoretical calculations fully reproduce the experimental results.

In summary, bound exciton-induced current and EL bistabilities with an S-type $I-V$ characteristic are observed in a silicon $p-n$-junction diode at temperatures below $30 \mathrm{~K}$. The two stable states in the current and the EL intensity from bound excitons and free excitons observed for a given voltage are correlated to the empty and occupied bound exciton states in the silicon $p-n$ diode. The consistency between the theoretical and experimental results indicates that bound excitons, despite their neutral-charged states, contribute to the $S$-type differential resistance in silicon $p-n$-junction diode.
We would like to thank I. Beatus, I. Winkler, S. Sinning, T. Gebel, and U. Lucchesi for assistance in the sample preparation and EL experiments, and U. Hornauer for fruitful discussions.

${ }^{1}$ A. Wacker and E. Schöll, J. Appl. Phys. 78, 7352 (1995).

${ }^{2}$ T. K. Carns, X. Zheng, and K. L. Wang, IEEE Electron Device Lett. 16, 256 (1995).

${ }^{3}$ W. C. Liu, J. H. Tsai, E. S. Lour, L. W. Laih, S. Y. Cheng, K. B. Thei, and C. Z. Wu, IEEE Trans. Electron Devices 44, 520 (1997).

${ }^{4}$ A. A. Lakhani and R. C. Potter, Appl. Phys. Lett. 52, 1684 (1988).

${ }^{5}$ A. Krotkus, A. Reklaitis, and A. Geizutis, J. Appl. Phys. 84, 3980 (1998).

${ }^{6}$ E. R. Brown, J. R. Söderström, C. D. Parker, L. J. Mahoney, K. M. Molvar, and T. C. Mc Gill, Appl. Phys. Lett. 58, 2291 (1991).

${ }^{7}$ R. P. Khosla, J. R. Fischer, and B. C. Burkey, Phys. Rev. B 7, 2551 (1973).

${ }^{8}$ E. Schöll, Z. Phys. B: Condens. Matter 46, 23 (1982).

${ }^{9}$ T. Yamamoto and M. Morimoto, Appl. Phys. Lett. 20, 269 (1972).

${ }^{10}$ J. Kastrup, H. T. Grahn, K. Ploog, F. Prengel, A. Wacker, and E. Schöll, Appl. Phys. Lett. 65, 1808 (1994).

${ }^{11}$ A. Zaslavsky, V. J. Goldman, D. C. Tsui, and J. E. Cunningham, Appl. Phys. Lett. 53, 1408 (1988).

${ }^{12}$ T. J. Foster, M. L. Leadbeater, L. Eaves, M. Henini, O. H. Hughes, C. A. Payling, F. W. Sheard, P. E. Simmonds, G. A. Toombs, G. Hill, and M. A. Pate, Phys. Rev. B 39, 6205 (1989).

${ }^{13}$ P. A. Parlangeli, P. C. M. Christianen, J. C. Maan, and M. Henini, Phys. Rev. B 63, 115307 (2001).

${ }^{14}$ Wal Lek Ng, M. A. Lourenco, R. M. Gwillia, S. Ledain, G. Shao, and K. P. Homewood, Nature (London) 410, 192 (2001)

${ }^{15}$ J.-P. Noël, N. L. Rowell, and J. E. Greene, J. Appl. Phys. 77, 4623 (1995).

${ }^{16}$ H. Weman, B. Monemar, G. S. Oehrlein, and S. J. Jeng, Phys. Rev. B 42, 3109 (1990).

${ }^{17}$ X. Y. Zhang, K. Dou, Q. Hong, and M. Balkanski, Phys. Rev. B 41, 1376 (1990). 